\title{
MIMO Geometry and Antenna Design for High Capacity and Improved Coverage in $\mathbf{m m}$-Wave Systems
}

\author{
Tommaso Cella, ${ }^{1}$ Pål Orten, ${ }^{2}$ and Jens Hjelmstad ${ }^{3}$ \\ ${ }^{1}$ NTNU and UniK, 7491 Trondheim, Norway \\ ${ }^{2} A B B$ and UniK, 1396 Billingstad, Norway \\ ${ }^{3}$ NTNU, 7491 Trondheim, Norway \\ Correspondence should be addressed to Tommaso Cella; tommaso@unik.no
}

Received 28 February 2013; Accepted 11 September 2013

Academic Editor: Yuan Yao

Copyright (C) 2013 Tommaso Cella et al. This is an open access article distributed under the Creative Commons Attribution License, which permits unrestricted use, distribution, and reproduction in any medium, provided the original work is properly cited.

\begin{abstract}
We show a way to optimize the capacity and at the same time achieve high coverage in LOS for a mm-wave system indoor. We optimize MIMO with regard to maximum Shannon capacity for a pure LOS channel. We describe the general procedure in order to maximize the capacity for our considered geometry, which consists of a circular arc array at the transmitter and a uniform linear array (ULA) at the receiver. The method is based on the optimization of the interelement distances at the transmitter and the receiver. High coverage is obtained with the use of the circular geometry and beamforming. We propose an example mm-wave system in the $70 \mathrm{GHz}$ portion of the E-band (71-76) GHz. The results show that the proposed system is able to achieve full coverage in LOS as well as high capacity, with practical dimensions.
\end{abstract}

\section{Introduction}

During the last years, there has been an increased interest in $\mathrm{mm}$-wave communications. The demand for fast data rate had a crucial role, and communication systems in the $\mathrm{mm}$-wave bands have been intensively investigated $[1,2]$. Although mm-wave extends from $30 \mathrm{GHz}$ to $300 \mathrm{GHz}$, with a resultant wavelength from $10 \mathrm{~mm}$ to $1 \mathrm{~mm}$, we commonly refer to fewer bandwidths, which include the V-band (57$66 \mathrm{GHz})$, the E-band $(71-76 \mathrm{GHz}$ and $81-86 \mathrm{GHz})$, and the W-band (92-95 GHz). Millimeter-wave wireless technologies provide higher data rates which are comparable to that of fiber optics but are less costly and easy to set up. The propagation characteristics at those frequencies are different compared to the lower ones, both in indoor and outdoor environments. While outdoor, the main sources of attenuation are due to atmospheric oxygen, humidity, fog, and rain [3]; indoor the signal experiences very high wall attenuation. In previous studies, in fact, it was shown that communications at mmwave bands are mainly LOS $[4,5]$. This is due not only to high attenuation, but also typically narrow antenna beams. A well-known method to improve the system capacity is the use of MIMO [2]. With the use of MIMO, communication links take advantage of multiplexing gain, because different information streams are sent from different transmitters towards different receivers at the same frequency. In order to get spatial multiplexing at lower frequencies, rich multipath is needed. The main advantage of using MIMO at $\mathrm{mm}$ wave bands is that by having a proper interelement spacing between transmitting and receiving antennas, multiple streams, and thus high capacity, can be obtained, even in LOS [6]. The capacity of LOS MIMO channels has been studied by several authors $[7,8]$. Different prototypes using mm-wave LOS MIMO were already developed $[9,10]$. Indoor MIMO channels at $5 \mathrm{GHz}$ and $60 \mathrm{GHz}$ were modeled and compared $[11,12]$. A further advantage of mm-wave MIMO systems at those bandwidths is that highly directive transmission and reception with electronically steerable beams can be achieved, using compact antenna arrays. Beamforming is then another practical way to improve the performance.

Our work is focused on guaranteeing two important requirements for $\mathrm{mm}$-wave wireless communications: provide high capacity and full LOS coverage, and we consider an indoor scenario. As mentioned before, a way to maximize 
the capacity in MIMO systems is to adjust the interelement distances at the transmitter and the receiver. A closed-form expression for the geometry maximizing capacity was found for the case of two uniform linear arrays (ULAs) in [6]. We consider a slightly different geometry, where the transmitter is a circular arc array, while the receiver is a ULA [13]. The rationale for this geometry will be explained later. An expression describing the geometry which maximizes the capacity in this case is derived in Section 3 of this paper. Applying this configuration, together with the use of beamforming, makes it possible for the receiver to be reached everywhere in LOS indoor. This would not be possible for the case of two ULAs, as will be described later in the paper. In our proposal, each MIMO element at the transmitter is itself a subarray, which can electronically scan the beam towards the receiver. The transmitter can then be considered an array of subarrays, in which each subarray represents an element of the MIMO system. The concept of array of subarrays was already investigated considering outdoor mm-wave links [14].

The rest of the paper is organized as follows: in Section 2 the capacity of MIMO systems is described; Section 3 is dedicated to the MIMO channel model and will focus on the geometry we introduce. In Section 4, an example mm-wave system is presented, while simulation results are shown in Section 5. Finally, the paper is concluded.

\section{Capacity of MIMO Systems}

A MIMO transmission system employs a number of transmit and receive antennas to transmit data over a channel. We denote the number of transmit antennas by $N$ and the number of receive antennas by $M$. Assuming slowly varying and frequency flat fading channels, we can model the MIMO transmission in complex baseband as [15]

$$
\mathbf{r}=\mathbf{H s}+\mathbf{n},
$$

where $\mathbf{r}$ is the $M \times 1$ received complex-valuated signal vector, $\mathbf{s}$ is the $N \times 1$ transmitted complex-valued signal vector, $\mathbf{H}$ is the $M \times N$ complex-valued channel matrix, and $\mathbf{n}$ is the $M \times 1$ complex-valued additive white Gaussian noise (AWGN) vector.

The additive noise vector contains i.i.d. circularly symmetric complex Gaussian elements with zero mean and variance $\sigma_{n}^{2}$, denoted $\mathrm{CN}\left(0, \sigma_{n}^{2}\right)$.

We denote the covariance matrix of the transmitted signal by $\mathbf{Q}=E\left[\mathbf{s s}^{H}\right]$. In practical systems, we usually need to fulfill an average transmit power constraint over the array.

If the total average transmit power is limited to $P_{T}$, then trace $(\mathbf{Q}) \leqslant P_{T}$ must be fulfilled. In the remainder of this paper, we will look at uncorrelated branch sources with equal power; that is, $\mathbf{Q}=\left(P_{T} / N\right) \mathbf{I}_{N}$. This is optimal with regard to capacity when $\mathbf{H}$ is unknown at the transmitter [2]. When such sources are used, the channel capacity of a MIMO system described by (1) becomes [6]

$$
C=\log _{2}\left[\operatorname{det}\left(\mathbf{I}_{M}+\frac{P_{T}}{N \sigma_{n}^{2}} \mathbf{H H}^{H}\right)\right] \text { bits } / \mathrm{s} / \mathrm{Hz},
$$

where $\mathbf{H}^{H}$ is the Hermitian transpose of the $\mathbf{H}$ matrix.
In the following, we also assume that all receiver antennas experience the same average received power. This average received power, $P_{R}$, is a function of the transmit power, $P_{T}$, the path loss, and the shadowing. The average received signalto-noise ratio (SNR) at one receive antenna then becomes $\bar{\gamma}=P_{R} / \sigma_{n}^{2}$. In the remainder of this paper, $\mathbf{H}$ is taken to be the normalized channel matrix, which implies that each element in $\mathbf{H}$ has unit average power. By requiring this normalization we make the average SNR independent of $\mathbf{H}$.

For convenience, we define the variables $U=\min (M, N)$ and $V=\max (M, N)$ for use in the rest of the paper. By doing an eigenvalue decomposition and using the previously mentioned expression for average received SNR, (2) can be written as [16].

$$
C=\sum_{i=1}^{U} \log _{2}\left(1+\frac{\bar{\gamma}}{N} \lambda_{i}\right) \text { bits } / \mathrm{s} / \mathrm{Hz}
$$

Here, $\lambda_{i}$ is the $i$ th eigenvalue of $\mathbf{W}$, which is defined as

$$
\mathbf{W}= \begin{cases}\mathbf{H H}^{H}, & M \leq N \\ \mathbf{H}^{H} \mathbf{H}, & M \geq N .\end{cases}
$$

Equation (3) shows that a MIMO system can be viewed as consisting of $U$ parallel single-input-single-output (SISO) channels, where each channel has gain $\lambda_{i}$, and an average SNR downscaled with the number of transmitters compared to a SISO system with the same total transmit power.

\section{MIMO Channel Model}

It is common practice to model the channel matrix as a sum of two components, a LOS component and a NLOS component.

The mm-wave system analyzed in this paper focuses only on the LOS channel because of the frequency band applied, as explained in the introduction. The entries in the LOS component matrix are discussed in more detail in the following section.

3.1. LOS Channel: Ray Tracing. We focus on the LOS Channel, and it has previously been demonstrated that, in order to optimize the MIMO capacity, the antennas must be properly spaced [6]. This is because a proper positioning of the antennas leads to a high-rank LOS channel matrix, corresponding to many nonzero eigenvalues $\lambda_{i}$. A closed form for two ULAs with arbitrary orientation was already found [6]. We derive an expression for the case when the transmitter consists of a circular arc array, while the receiver is a ULA, as in Figure 1. The design criteria to improve the LOS capacity will focus on the antenna separation at the receiver, while the transmitter is fixed. With this geometry, as will be shown later in this paper, it is possible to achieve full coverage in LOS.

Referring to Figure 1, the transmitting antenna consists of equally spaced elements placed on a circular arc of total aperture equal to $2 \alpha$. The receiver is a ULA. The interantenna distances $d_{t}$ and $d_{r}$, at the transmitter and receiver, respectively, are constant. In Figure 1 , the $x$-axis taken to be 


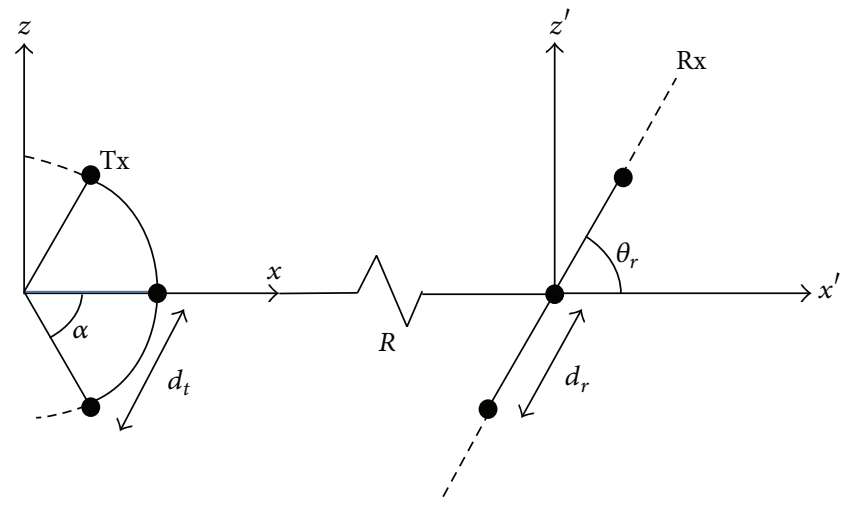

FIGURE 1: Geometry of the proposed system.

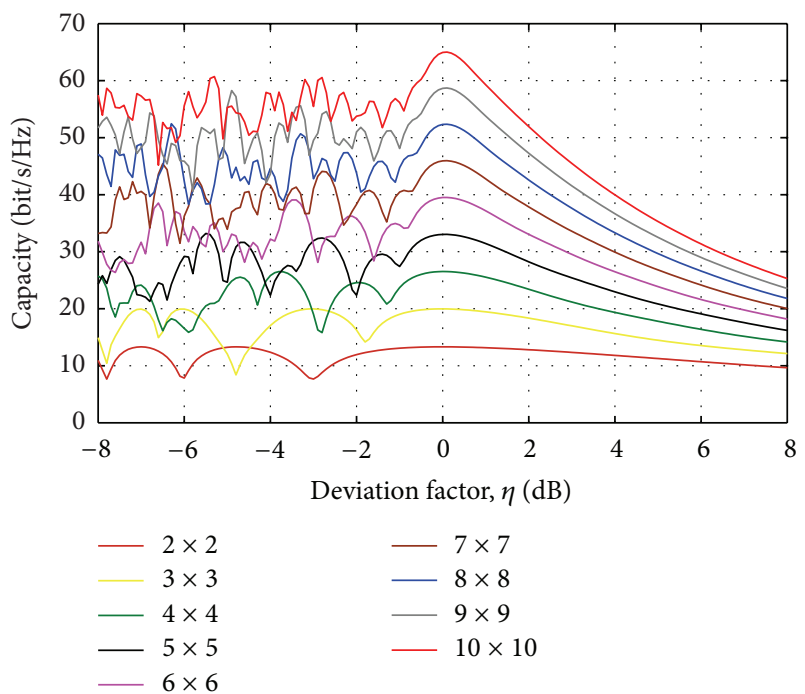

FIGURE 2: LOS capacity by varying the number of transmitters $(N=$ $M)$, for different values of the deviation factor $\eta(\mathrm{dB})$.

in the direction from the center of the circular arc which includes the transmit array, to the center of the receiving array. Both the arrays are placed in the $x z$-plane. The distance between the origin and the receiver is denoted by $R$. The total length of the transmitter is approximated to $(N-1) \cdot d_{t}$, and the total length of the receiver is $(M-1) \cdot d_{r}$. The radius of the circle which includes the transmitter is denoted by $r$. Due to the constant distance between elements in the transmitter, it can be approximated by

$$
r \approx \frac{d_{t}(N-1)}{2 \alpha} .
$$

The angles of the local spherical coordinate system at the transmitter and receiver are denoted by $\theta_{t}$ and $\theta_{r}$.

In order to make $\mathbf{H}_{\mathrm{LOS}}$ a high-rank channel matrix, we need to choose appropriate values for $d_{t}$ and $d_{r}$. The technique used to find such values is based on ray tracing.

The first step is to define the vectors $\mathbf{a}_{n}^{t}$ and $\mathbf{a}_{m}^{r}$, from the origin to the transmit and receive antenna elements $(n$ and $m)$, respectively (the elements are ordered from $-(N-1) / 2$

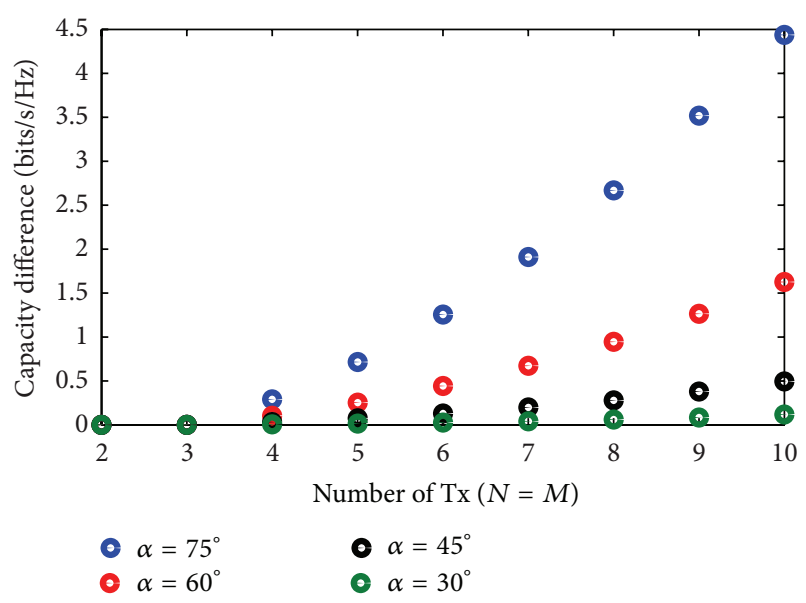

FIGURE 3: Difference between the maximum theoretical capacity and the actual achieved capacity by varying the number of transmitters $(N=M)$ and the arc aperture $\alpha$.

to $(N-1) / 2$ and $-(M-1) / 2$ to $(M-1) / 2$ at the lower end of the arrays). From Figure 1 we have

$$
\begin{gathered}
\mathbf{a}_{n}^{t}=r \sin \left[\alpha \frac{n}{(N-1) / 2}\right] \mathbf{n}_{z}+r \cos \left[\alpha \frac{n}{(N-1) / 2}\right] \mathbf{n}_{x}, \\
n \in\left\{-\frac{N-1}{2}, \ldots, \frac{N-1}{2}\right\}, \\
\mathbf{a}_{m}^{r}=m d_{r} \sin \theta_{r} \mathbf{n}_{z}+R+m d_{r} \cos \theta_{r} \mathbf{n}_{x}, \\
m \in\left\{-\frac{M-1}{2}, \ldots, \frac{M-1}{2}\right\},
\end{gathered}
$$

where $\mathbf{n}_{x}$ and $\mathbf{n}_{z}$ are the unit vectors in their respective directions. The path length $r_{m, n}$, between transmit antenna $n$ and receive antenna $m$, can be found by calculating the Euclidean norm of the vector difference between $\mathbf{a}_{m}^{r}$ and $\mathbf{a}_{n}^{t}$ :

$$
\begin{aligned}
r_{m, n}= & \left\|\mathbf{a}_{m}^{r}-\mathbf{a}_{n}^{t}\right\| \\
= & {\left[\left(R+m d_{r} \cos \theta_{r}-r \cos \left[\alpha \frac{n}{(N-1) / 2}\right]\right)^{2}\right.} \\
& \left.+\left(m d_{r} \sin \theta_{r}-r \sin \left[\alpha \frac{n}{(N-1) / 2}\right]\right)^{2}\right]^{1 / 2} \\
r_{m, n} \approx & R-r+\frac{\left(m d_{r} \sin \theta_{r}-r \alpha(n /((N-1) / 2))\right)^{2}}{2(R-r)} .
\end{aligned}
$$

The final expression was derived considering some simplifications. We consider the case where $\theta_{r}$ is close to $90^{\circ}$ and thus $\cos \theta_{r}$ is close to 0 . The distance between the transmit elements and the origin in the $x$-axes was set to be $r$, while only the argument of the sin was considered in the $z$-axes. A better approximation for the sin will be taken into account later in this section. We consider the scenario in which $R \gg r$. To simplify the square root, the following expression was used: $\sqrt{x^{2}+a} \approx x+a / 2 x$. 


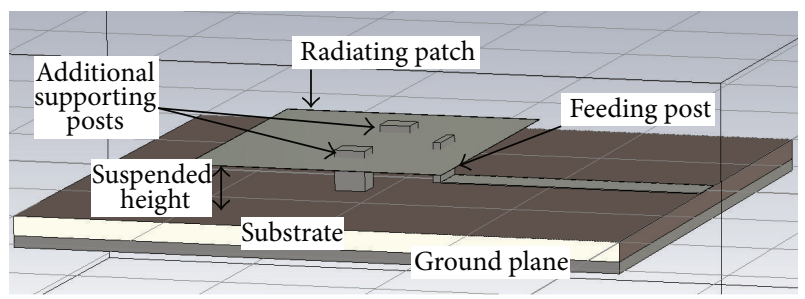

FIgURE 4: Single element suspended patch antenna.

TABLE 1: Example link budget parameters.

\begin{tabular}{lcc}
\hline Tx Power & 3 & $\mathrm{dBm}$ \\
GTx & 19 & $\mathrm{dBi}$ \\
GRx & 10 & $\mathrm{dBi}$ \\
Path loss [4] & -91 & $\mathrm{~dB}$ \\
Background noise & -174 & $\mathrm{dBm} / \mathrm{Hz}$ \\
Noise BW & 93 & $\mathrm{~dB}$ \\
Noise figure Rx & 5 & $\mathrm{~dB}$ \\
\hline SNR Rx & $\mathbf{1 7}$ & $\mathrm{dB}$ \\
\hline
\end{tabular}

The symbols transmitted from each antenna at the transmitter will be received at $M$ receive antennas and are subjected to a path length $r_{m, n}$ as given by (8) and an interantenna phase difference. The received vector from transmit antenna $n$ on the $M$ receive antennas is then

$$
h_{n}=\left[\exp \left(\frac{j 2 \pi}{\lambda} r_{0, n}\right), \ldots, \exp \left(\frac{j 2 \pi}{\lambda} r_{M-1, n}\right)\right]^{T}
$$

in which $\lambda$ is the wavelength and $(\cdot)^{T}$ indicates the vector transpose. The channel matrix is then

$$
\mathbf{H}_{\mathrm{LOS}}=\left[\mathbf{h}_{0}, \mathbf{h}_{1}, \ldots, \mathbf{h}_{\mathrm{N}-1}\right] .
$$

We are going to derive the antenna separation which maximizes the Shannon capacity by considering a pure LOS channel. We Consider that trace $(\mathbf{W})=\sum_{i=1}^{U} \lambda_{i}$ and that the trace of $\mathbf{W}$ for a pure LOS channel matrix can be shown to be $N \cdot M(=V \cdot U)$. Using the method of Lagrange multipliers and maximizing (3), the optimal solution is achieved when all eigenvalues are equal (i.e., $\lambda_{1}=\lambda_{2}=\cdots=\lambda_{U}=V$ ). One realization of $\mathbf{W}$ that fulfills this requirement is

$$
\mathbf{W}=V \cdot \mathbf{I}_{U \times U},
$$

where $\mathbf{I}_{U \times U}$ is the $U \times U$ identity matrix. We therefore need to get $\mathbf{H}_{\mathrm{LOS}}$ to have orthogonal columns for $M$ larger than $N$, or orthogonal rows, for $N$ larger than $M$. We consider the situation where $M$ is larger than $N$. Orthogonality between the different columns is obtained if the inner product of two received vectors from two different transmit antennas is equal to zero for all combinations of $k, l$ :

$$
\begin{aligned}
\left\langle h_{k}, h_{l}\right\rangle & =\sum_{m=0}^{M-1} \exp \left(j \frac{2 \pi}{\lambda}\left(r_{m, k}-r_{m, l}\right)\right) \\
& =\sum_{m=0}^{M-1} \exp \left(j 2 \pi \frac{d_{r} \sin \theta_{r} r(\alpha /((N-1) / 2))(k-l) m}{\lambda(R-r)}\right) .
\end{aligned}
$$

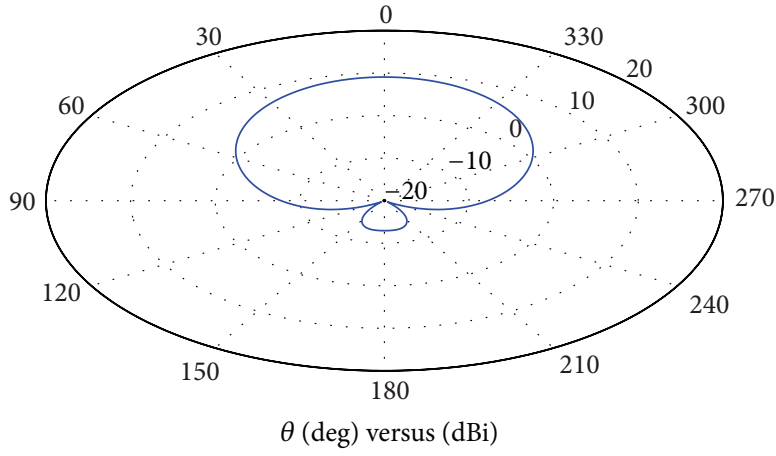

Frequency $=73 \mathrm{GHz}$

Main lobe magnitude $=9.1 \mathrm{~dB}$

Main lobe direction $=0 \mathrm{deg}$

Angular width $(3 \mathrm{~dB})=66.4 \mathrm{deg}$

Side lobe level $=-22 \mathrm{~dB}$

(a)

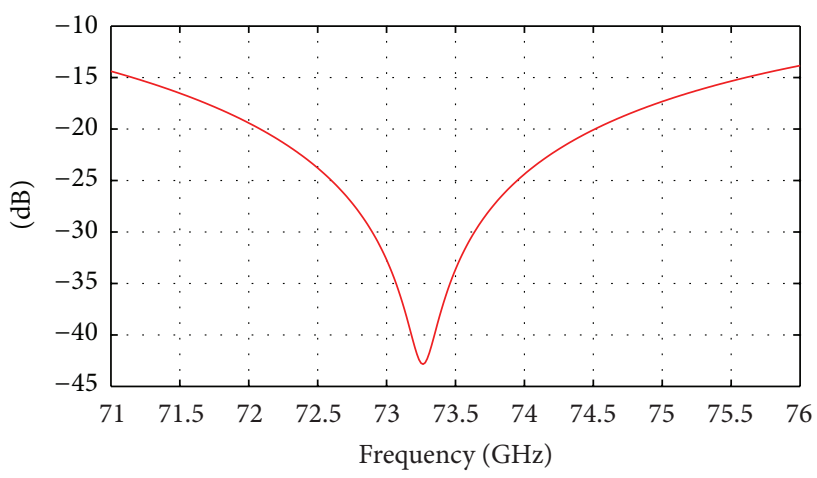

(b)

Figure 5: Designed patch performances: (a) radiation pattern (b) reflection coefficient $(\mathrm{dB})$.

Now using the expression for finite geometric series, we get

$$
\begin{aligned}
& \left\langle h_{k}, h_{l}\right\rangle \\
& \quad=\frac{\sin \left(\pi\left(d_{r} / \lambda(R-r)\right) \sin \theta_{r}(\alpha /((N-1) / 2))(k-l) M\right)}{\sin \left(\pi\left(d_{r} / \lambda(R-r)\right) \sin \theta_{r}(\alpha /((N-1) / 2))(k-l)\right)} \\
& \quad=0 .
\end{aligned}
$$

This can further be expressed as

$$
d_{r}=\frac{((N-1) / 2)\left(\lambda(R-r) / \alpha r \sin \theta_{r}\right)}{M} .
$$

As we can see, there are different solutions, but we chose the smallest antenna distance, which is usually preferred.

Now considering that $r \approx d t(N-1) /(2 \alpha)$, we have

$$
d_{r}=\frac{\left(\lambda(R-r) / d_{t} \sin \theta_{r}\right)}{M} .
$$

Unless the maximum arc aperture is below $30^{\circ}$, a much better expression is found if we multiply this result by $\alpha / \sin \alpha$. 


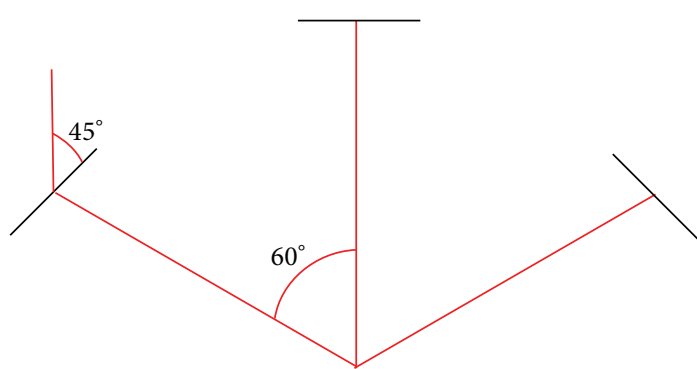

Frequency $=73 \mathrm{GHz}$

Main lobe magnitude $=20.4 \mathrm{~dB}$ Angular width $(3 \mathrm{~dB})=4.7 \mathrm{deg}$

Side lobe level $=-20.4 \mathrm{~dB}$ (a)

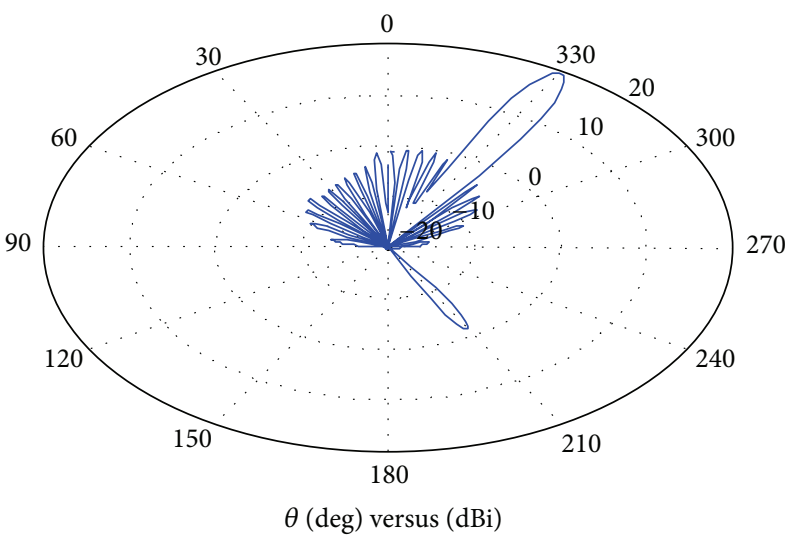

Frequency $=73 \mathrm{GHz}$

Main lobe magnitude $=19.6 \mathrm{~dB}$

Main lobe direction $=-30 \mathrm{deg}$

Angular width $(3 \mathrm{~dB})=5.8 \mathrm{deg}$

Side lobe level $=-20 \mathrm{~dB}$
Main lobe direction $=0 \mathrm{deg}$

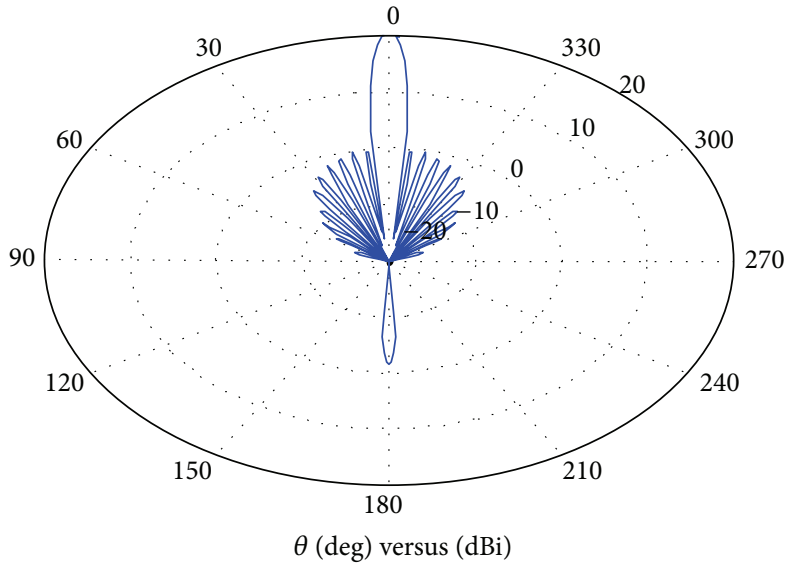

(b)

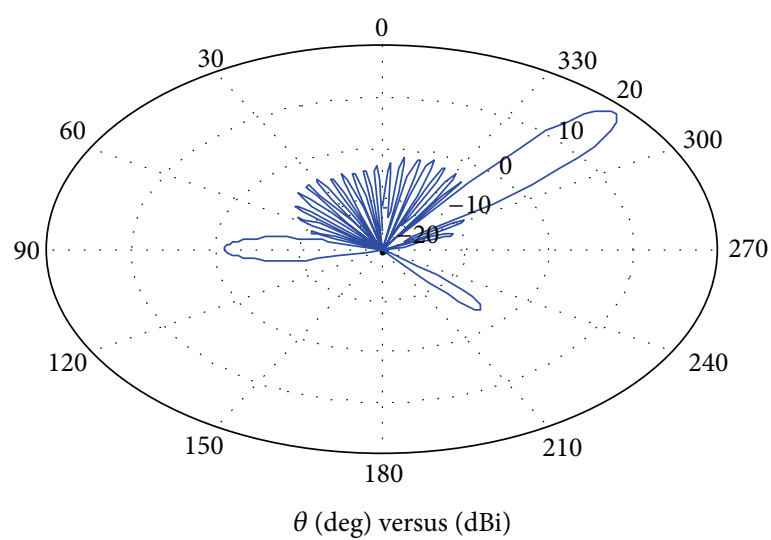

Frequency $=73 \mathrm{GHz}$

Main lobe magnitude $=18.8 \mathrm{~dB}$

Main lobe direction $=-45 \mathrm{deg}$

Angular width $(3 \mathrm{~dB})=7.4 \mathrm{deg}$

Side lobe level $=-20 \mathrm{~dB}$

(d)

FiguRE 6: Transmitter geometry (a) and subarray radiation patterns for different focusing angles in azimuth: broadside (b) $30^{\circ}$ (c) and $45^{\circ}$ (d).

This is because otherwise the small angle approximation we adopted to get to (8) is inaccurate. We then finally obtain

$$
d_{r}=\frac{\left(\lambda(R-r) / d_{t} \sin \theta_{r}\right)}{M} \frac{\alpha}{\sin \alpha} .
$$

From (16), we can see that the distance between receiving antenna elements depend on the wavelength, transmitterreceiver distance, the number of receive antennas, the transmit array parameters $d_{t}, r$, and $\alpha$, and $\theta_{r}$.

What comes from (16) is that the optimum $d_{r}$ is calculated for a fixed distance between transmitter and receiver. We will show later the effects of a varying distance $R$. In order to show the effectiveness of (16), we now define a deviation factor $\eta$ similar to [6], set as the ratio between the optimal $d_{r}$ and the actual $d_{r}$ :

$$
\eta=\frac{d_{\text {ropt }}}{d_{r}} .
$$

We show in Figure 2 the LOS capacity calculated with (3) for SNR set to $20 \mathrm{~dB}$. The figure considers $N$ varying from 2 to 10 and $N=M$. The frequency was set to $73 \mathrm{GHz}$ and the maximum arc aperture $2 \alpha=120^{\circ}$, while $R$ is $5 \mathrm{~m}$. We also assume that $d_{t}=10 \mathrm{~cm}$ (corresponding to $\approx 25 \lambda$ ) and $\theta_{r}=90^{\circ}$. Analyzing the figure, we can observe that there is a peak for the deviation factor equal to $0 \mathrm{~dB}$, or to a value very close to that. The peaks in Figure 2 correspond 


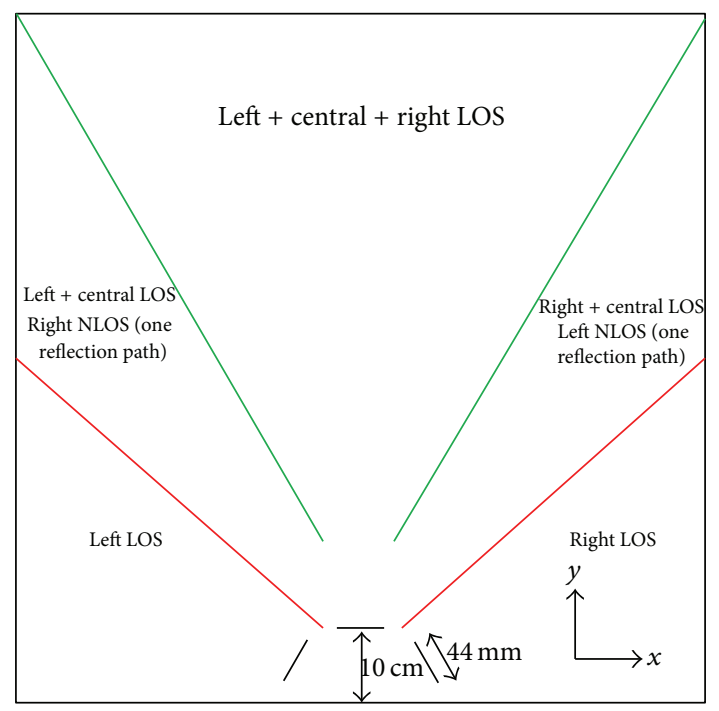

FIGURE 7: Coverage areas for our system.

to the smallest distance $d_{r}$ able to maximize the capacity. Due to the approximations adopted, they do not correspond exactly to the maximum theoretical achievable capacity by using (3). The difference between the maximum and the achieved capacity is shown in Figure 3 . In the figure we also show the effect on the capacity by varying the arc aperture at the transmitter. As we can see, this difference increases when the number of elements increases and when the arc aperture is wider. Next section is focused in the design of a system with a suitable geometry that according to (16) is able to achieve high capacity and full LOS coverage indoor.

\section{Example MM-Wave System}

From a mm-wave system design point of view, an obvious goal is to maximize the capacity and at the same time achieve full coverage in LOS. Our system is assumed to operate in the $70 \mathrm{GHz}$ portion of the E-band $(71-76 \mathrm{GHz})$ indoor (Same design procedure could be applied to all $\mathrm{mm}$-wave frequencies (like $60 \mathrm{GHz}$ )). As described before, it is crucial at those frequencies to achieve LOS links, due to the high losses.

The system proposed is a $3 \times 3$ MIMO where the transmitter nodes correspond to a circular arc array and the receiver is a ULA as shown in Figure 1. The fundamental point of having this geometry is that we are able to achieve full LOS coverage in azimuth, with the use of beamforming. Each of the 3 elements of the transmitter is in fact a subarray, which by electronically switching the beam provides full LOS coverage as well as additional gain and low sidelobes. Reducing the sidelobe levels represents also a decisive aspect which will dramatically reduce the effects of multipath.

The system analyzed in the following sections will consider different aspects. First of all a baseline link budget is exploited; then the single element is designed. After that, the performances of the single element as well as the subarray farfield pattern will be described.
4.1. Example Link Budget. The link budget is described in Table 1. The following values are taken as examples and can vary according to the system requirements. The central frequency considered is $73 \mathrm{GHz}$. The total transmit power is $9 \mathrm{dBm}$ and we assume equal power allocation as a first step. To compute the noise BW we consider a BW of $2 \mathrm{GHz}$. The path loss is calculated for a distance of $10 \mathrm{~m}$ according to [17]. As we can see, at a distance of $10 \mathrm{~m}$ we can achieve up to about $17 \mathrm{~dB}$ SNR at each receiver. With such SNR, we are able to consider different modulations, depending on the design projects.

4.2. Single Element and Array Design. For the proposed system, we use a suspended patch antenna. Such antenna achieves improved performance compared to conventional patch antennas in terms of gain and bandwidth. A similar kind of antenna was already developed and tested at the $77 \mathrm{GHz}$ automotive radar frequency [18]. The designed element is illustrated in Figure 4 and simulations were done using CST Microwave Studio.

The substrate has a permittivity equal to 2.2 , the suspended height is $200 \mu \mathrm{m}$, and the patch length and width are, respectively, $1.7 \mathrm{~mm}$ and $1.1 \mathrm{~mm}$. Two additional supporting posts are considered in order to sustain the radiating patch more robustly. Figure 5 shows the E-field pattern of the designed array and the reflection coefficient for the bandwidth of interest. As we can see, the gain is about $9 \mathrm{dBi}$ at $73 \mathrm{GHz}$, while the reflection coefficient is below $-15 \mathrm{~dB}$ for the all band of interest.

As already explained, each transmitter is itself a subarray. Using this concept, we are able to achieve the desired coverage by smartly placing the transmitter in a room. In Figure 1, each point represents a MIMO transmit antenna. Each transmit antenna is a half-wavelength spaced array, which electronically focuses the main beam towards the receive antenna.

Referring to Figure 1, we consider a $3 \times 3$ MIMO system where the transmitter arc aperture $2 \alpha$ is $120^{\circ}$. We want to find the interelement distance at the receiver to optimize the capacity according to (3). We set the transmitter receiver distance equal to $5 \mathrm{~m}$, the radius $r$ of the circle which constitutes the transmitter is $10 \mathrm{~cm}$ (corresponding to an interelement distance equal to $\approx 25 \lambda$ ) and $\theta_{r}$ equal to 0 . The calculated $d_{r}$ from (16) is then $d_{r} \approx 8 \mathrm{~cm}$. This makes it possible to have the receiver fit in for instance a laptop.

The performance of each subarray at the transmitter was simulated for different focusing angles in azimuth. The transmitter geometry and the radiation patterns are shown in Figure 6. Each subarray at the transmitter is a ULA with 20 elements in azimuth, which makes the subarray about $4.4 \mathrm{~cm}$ long. The transmitter elements are divided in left, central, and right subarray. The left and right subarrays are shifted $45^{\circ}$ compared to the central element, as shown in Figure 6.

The array is able to achieve a $\pm 50^{\circ}$ coverage in azimuth, and the gain is between $18.8 \mathrm{dBi}$ and $20.4 \mathrm{dBi}$ depending on the focusing angle. A taylor window is applied in order to decrease the side lobes to be at least $20 \mathrm{~dB}$ lower than the main lobe. This is important because in such a system they would contribute only to the multipath, which is not 


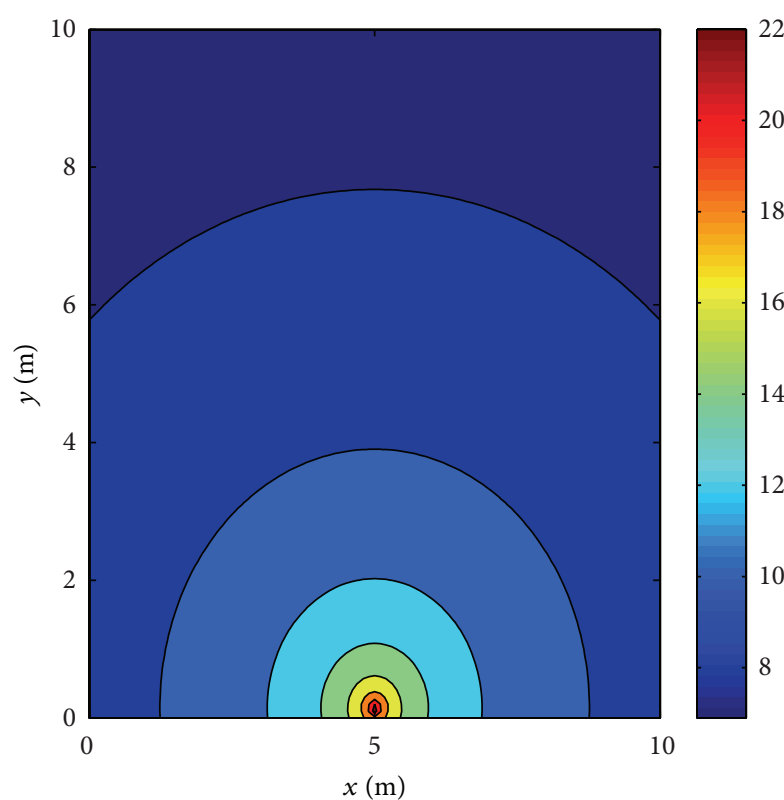

(a)

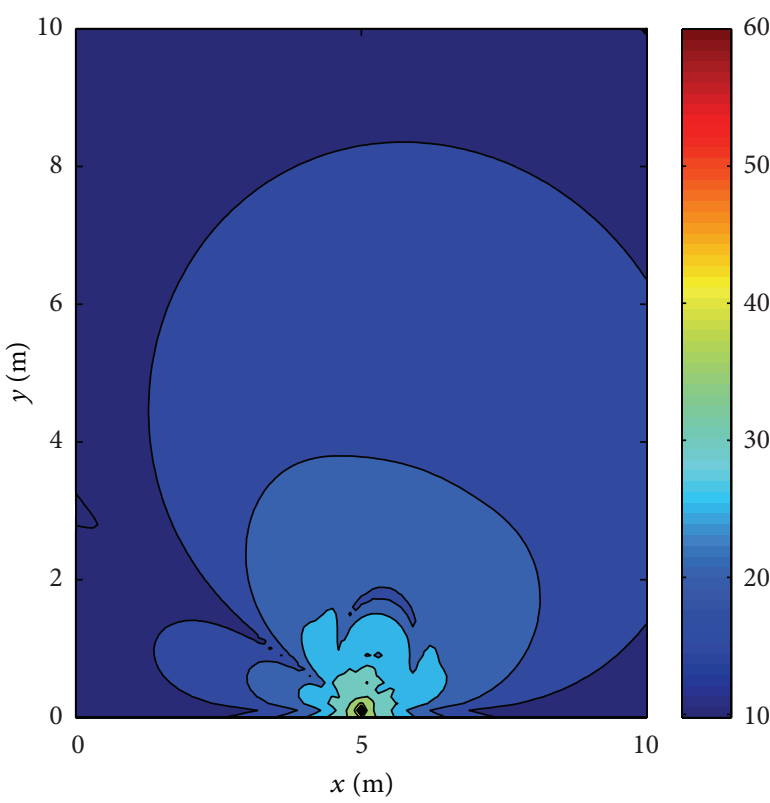

(c)

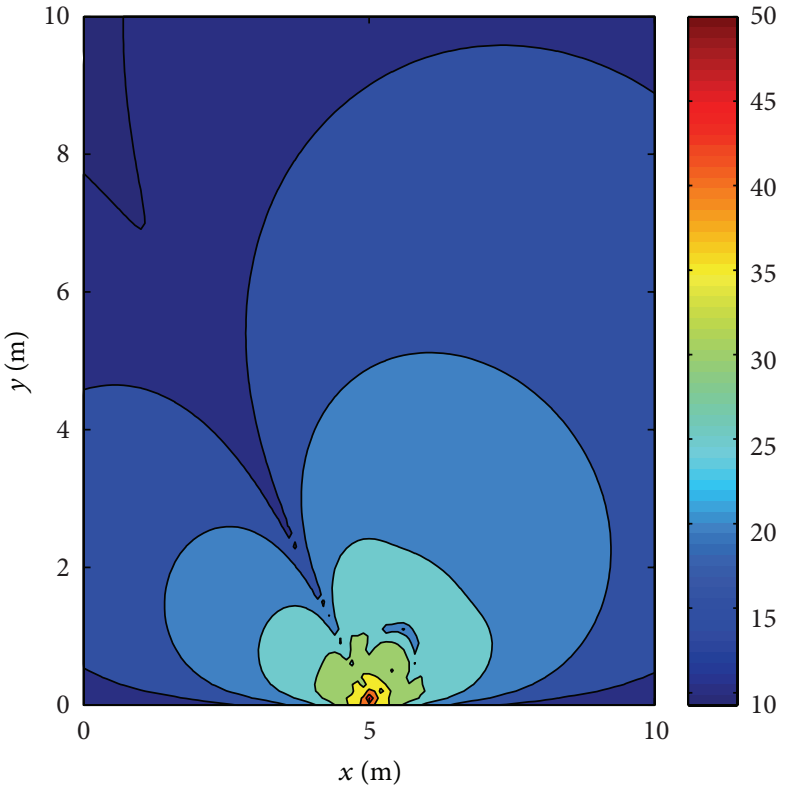

(b)

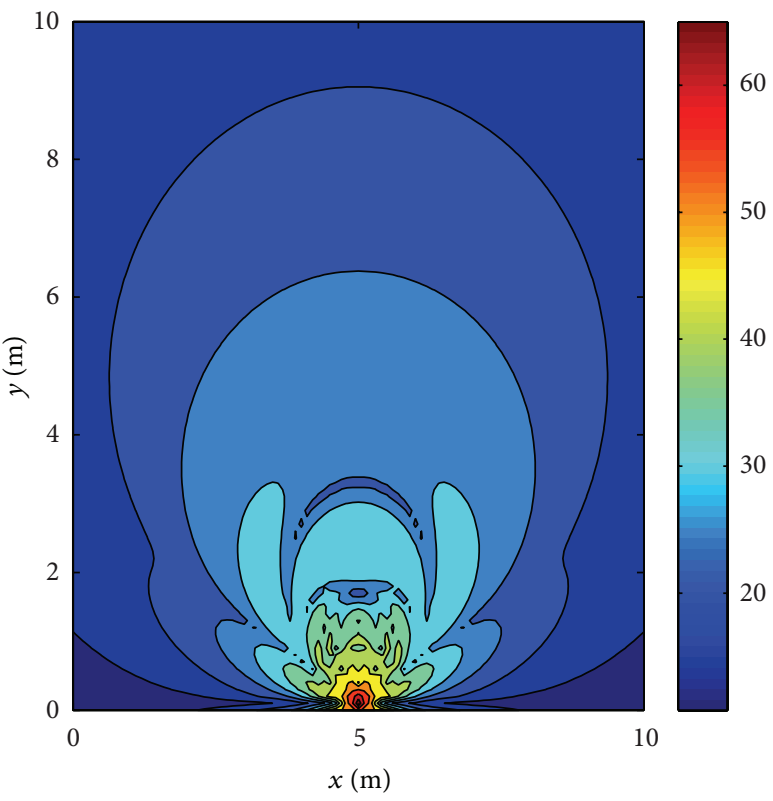

(d)

FIGURE 8: Indoor capacity (bit/s/Hz) achievable for different scenarios: (a) SIMO (b) $2 \times 3$ MIMO (c) central and right element in LOS, left element with one reflection (d) $3 \times 3$ MIMO in LOS.

desired for a mm-wave LOS MIMO system. With the use of a circular arc array at the transmitter, we are able to achieve full hemispherical coverage with relatively narrow beamwidths. This would not be possible in case of a linear array, since the typical maximum coverage, attainable with patch elements, is $\pm 60^{\circ}$ in the broadside direction.

\section{Numerical Results}

This section will show the capacity achievable with the designed system indoor. We consider a $10 \times 10 \mathrm{~m}$ room, where the origin of the circle containing the transmitter is placed in coordinates $(5,0)$, as shown in Figure 8 , while the receiver is moving. We consider only the LOS component. In order to calculate the SNR in the receiver location, refer to the link budget parameters described previously. Considering that, with the use of beamforming and the geometry considered, each point in the room can be reached in LOS, we investigate 3 different situations: (i) the receiver is mainly reachable by only one subarray in LOS (area below the red lines in Figure 7), (ii) the receiver is reachable by 2 subarrays in LOS, eventually the 3 rd with a one reflection path (area between 


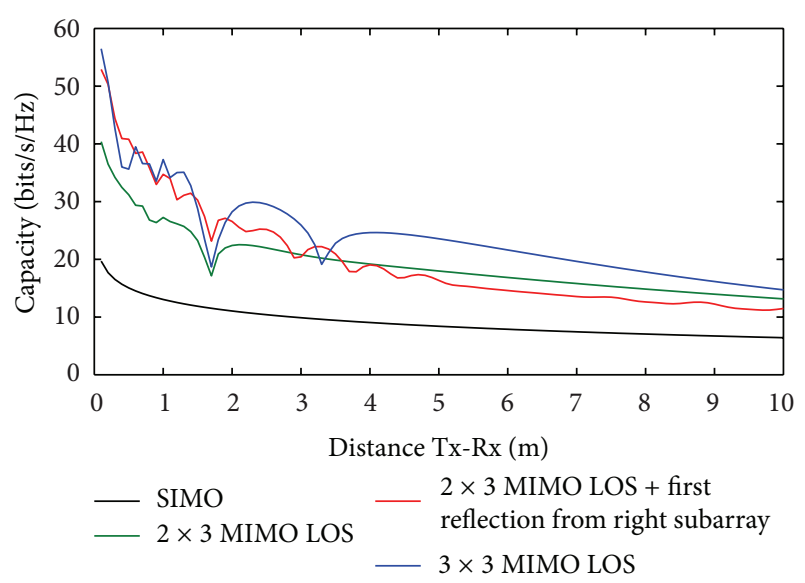

FIGURE 9: Indoor capacity for a moving receiver.

the red and green lines in Figure 7), and (iii) the receiver is reachable by 3 subarrays in LOS (area between the green lines in Figure 7).

The capacity attainable for the 3 scenarios is shown in Figure 8. Figure 8 (a) shows the capacity when the receiver is reached by only one transmit subarray, which corresponds to the capacity of a SIMO in LOS. Figure 8(b) shows the capacity achievable for a $2 \times 3 \mathrm{MIMO}$ in LOS. Here we consider only the central and right subarrays. Figure 8(c) considers the case where the receiver is reachable by the central and right subarrays in LOS, while the left subarray focuses its beam towards the receiver with a one reflection path. In Figure 8(d) we can see the capacity for a $3 \times 3$ MIMO in LOS.

In order to calculate the capacity for Figure 8(c), we modeled the walls as plasterboard and took the parameters from [4]. The channel matrix is calculated considering the additional path due to the reflection and the reflection coefficient for the material considered, as shown in [3]. What comes from Figure 8 is that depending on the location of the receiver, there is a huge difference in the maximum capacity achievable, while a minimum capacity of $8 \mathrm{bit} / \mathrm{s} / \mathrm{Hz}$ is always guaranteed in LOS. In addition, Figure 9 shows the capacity feasible for the proposed scenarios when the transmitter is fixed as before, and the receiver is moving along the $y$ direction, from $(5,0)$ towards $(5,10)$.

\section{Conclusion}

This paper proposed a mm-wave MIMO system able to achieve high-capacity as well as full-coverage indoor in LOS. This is possible by considering a particular geometry for the transmitter and receiver, which involves proper dimensioning of the interelement distances between each MIMO node. The geometry involves both linear and circular MIMO arrays, as well as subarrays. The proposed system considers only LOS links and highly directive beams with low side lobes. Such configuration is adopted in order to drastically reduce the multipath. Further research will be focused on evaluating the performances of the proposed system in a real scenario.

\section{References}

[1] A. Arvanitis, G. Anagnostou, N. Moraitis, and P. Constantinou, "Capacity study of a multiple element antenna configuration in an indoor wireless channel at $60 \mathrm{GHz}$," in Proceedings of the IEEE 65th Vehicular Technology Conference (VTC '07), pp. 609613, April 2007.

[2] D. Jie, J.-J. Wang, H. Zhang, and G.-Y. Wang, "Channel capacity of $60 \mathrm{GHz}$ wireless communication systems over indoor lineof-sight and non-line-of-sight channels," in Proceedings of the 6th International Conference on Wireless Communications, Networking and Mobile Computing (WiCOM '10), Chengdu, China, September 2010.

[3] "Specific attenuation model for rain for use in prediction methods," Recommendation ITU-R P.838-3, 2005.

[4] E. Torkildson, U. Madhow, and M. Rodwel, "On the feasibility of spatial multiplexing for indoor $60 \mathrm{GHz}$ communication," in Proceedings of the ACM International Workshop on mmWave Communications: From Circuits to Networks (mmCom '10), pp. 57-61, Chicago, Ill, USA, September 2010.

[5] E. Torkildson, U. Madhow, and M. Rodwell, "Indoor millimeter wave MIMO: feasibility and performance," IEEE Transactions on Wireless Communications, vol. 10, no. 12, pp. 4150-4160, 2011.

[6] F. Bøhagen, P. Orten, and G. E. Øien, "Design of optimal high-rank line-of-sight MIMO channels," IEEE Transactions on Wireless Communications, vol. 6, no. 4, pp. 1420-1424, 2007.

[7] I. Sarris and A. Nix, "Design and performance assessment of maximum capacity MIMO architectures in line-of-sight," IEE Proceedings, vol. 153, no. 4, pp. 482-488, 2006.

[8] D. Gesbert, H. Bölcskei, D. A. Gore, and A. J. Paulraj, "Outdoor MIMO wireless channels: models and performance prediction," IEEE Transactions on Communications, vol. 50, no. 12, pp. 19261934, 2002.

[9] C. Sheldon, M. Seo, E. Torkildson, U. Madhow, and M. Rodwell, "A $2.4 \mathrm{~Gb} / \mathrm{s}$ millimeter-wave link using adaptive spatial multiplexing," in Proceedings of the IEEE International Symposium on Antennas and Propagation (APSURSI '10), Toronto, Canada, July 2010.

[10] C. Sheldon, M. Seo, E. Torkildson, M. Rodwell, and U. Madhow, "Four-channel spatial multiplexing over a millimeter-wave lineof-sight link," in Proceedings of the IEEE MTT-S International Microwave Symposium (IMS '09), pp. 389-392, June 2009.

[11] S. Kirthiga and M. Jayakumar, "Performance and capacity analysis of MIMO system at $5 \mathrm{GHz}$ and $60 \mathrm{GHz}$ in indoor environment," WSEAS Transactions on Communications, vol. 11, no. 11, p. 415, 2011.

[12] S. Manojna, S. Kirthiga, and M. Jayakumar, "Spatial multiplexing for millimeter waves using TSV model," International Journal of Computer Science and Engineering Technology, vol. 1, no. 5, pp. 244-248, 2011.

[13] T. Cella and P. Orten, "Design of a MIMO geometry for high capacity and full coverage mm-wave system," in Proceedings of the 5th International Conference on Computers and Devices for Communication (CODEC '12), Kolkata, India, 2012.

[14] E. Torkildson, C. Sheldon, U. Madhow, and M. Rodwell, "Nonuniform array design for robust millimeter-wave MIMO links," in Proceedings of the IEEE Global Telecommunications Conference (GLOBECOM '09), Honolulu, Hawaii, USA, December 2009.

[15] D. Gesbert, M. Shafi, D.-S. Shiu, P. J. Smith, and A. Naguib, "From theory to practice: an overview of MIMO space-time 
coded wireless systems," IEEE Journal on Selected Areas in Communications, vol. 21, no. 3, pp. 281-302, 2003.

[16] E. Telatar, "Capacity of multiantenna gaussian channels," Technical Memos, AT\&T Bell Labratories, 1995.

[17] "Propagation data and prediction methods for the planning of indoor radiocommunication systems and radio local area networks in the frequency range $900 \mathrm{MHz}$ to $100 \mathrm{GHz}$," Recommendation ITU-R P.1238-7, 2012.

[18] H. S. Lee, J.-G. Kim, S. Hong, and J.-B. Yoon, "Micromachined $\mathrm{CPW}$-fed suspended patch antenna for $77 \mathrm{GHz}$ automotive radar applications," in Proceedings of the 8th European Conference on Wireless Technology (ECT '05), pp. 269-272, October 2005. 

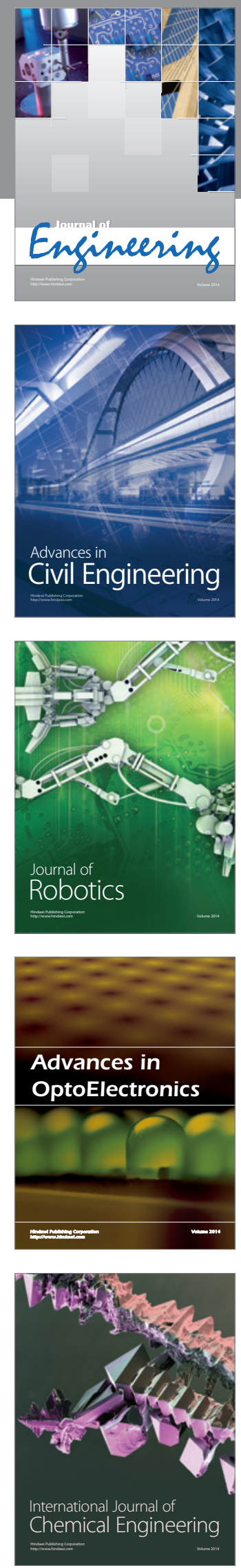

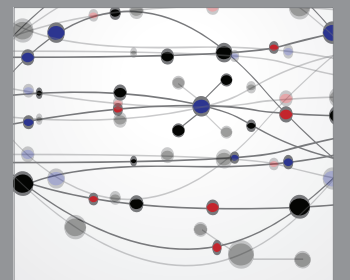

The Scientific World Journal
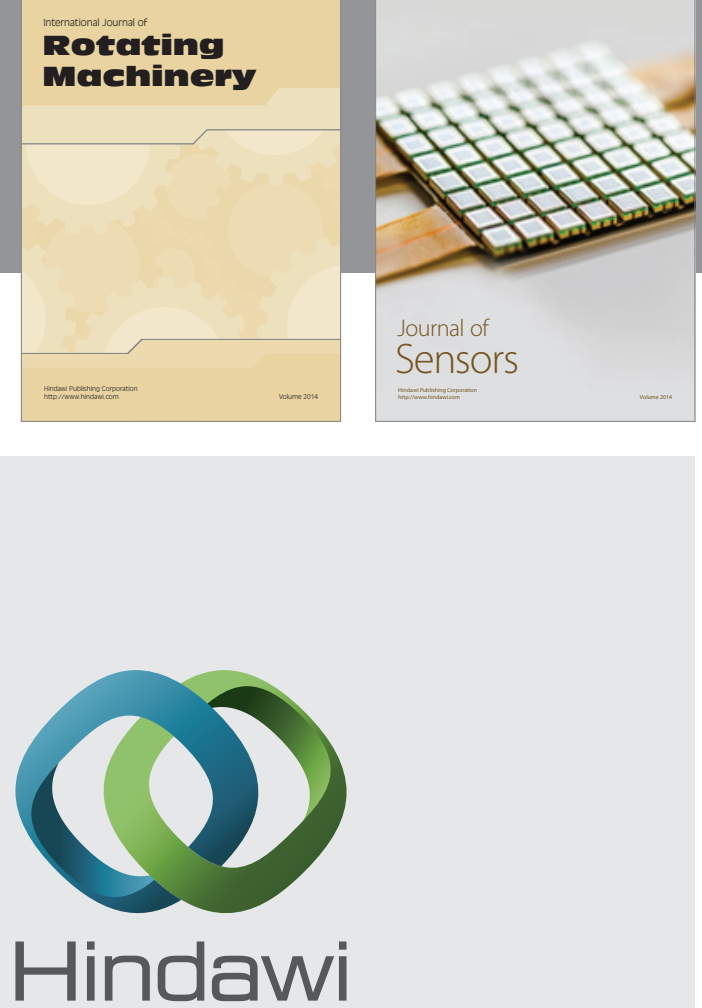

Submit your manuscripts at http://www.hindawi.com
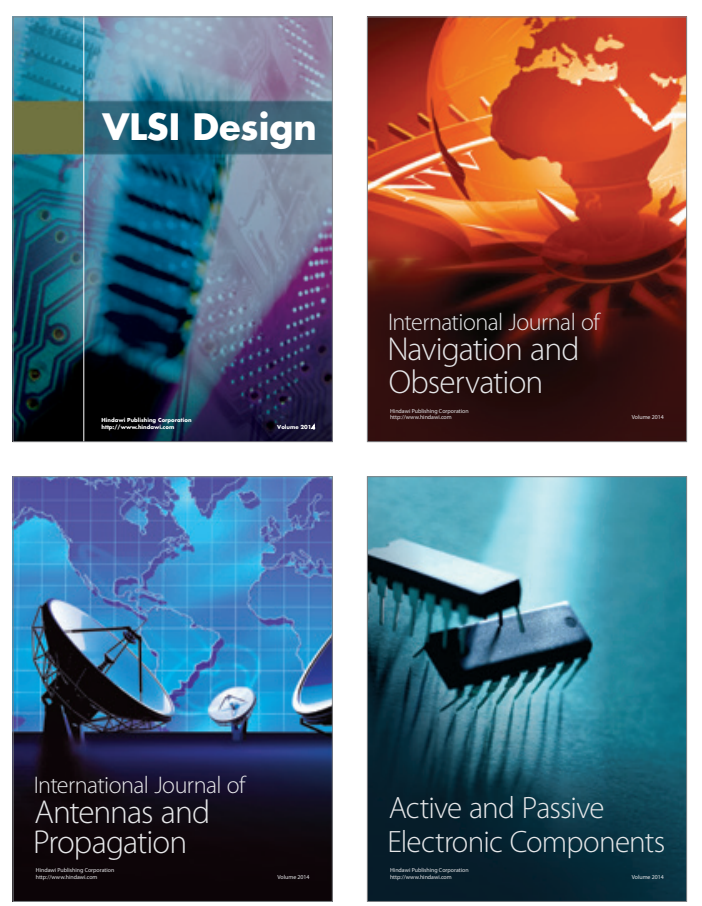
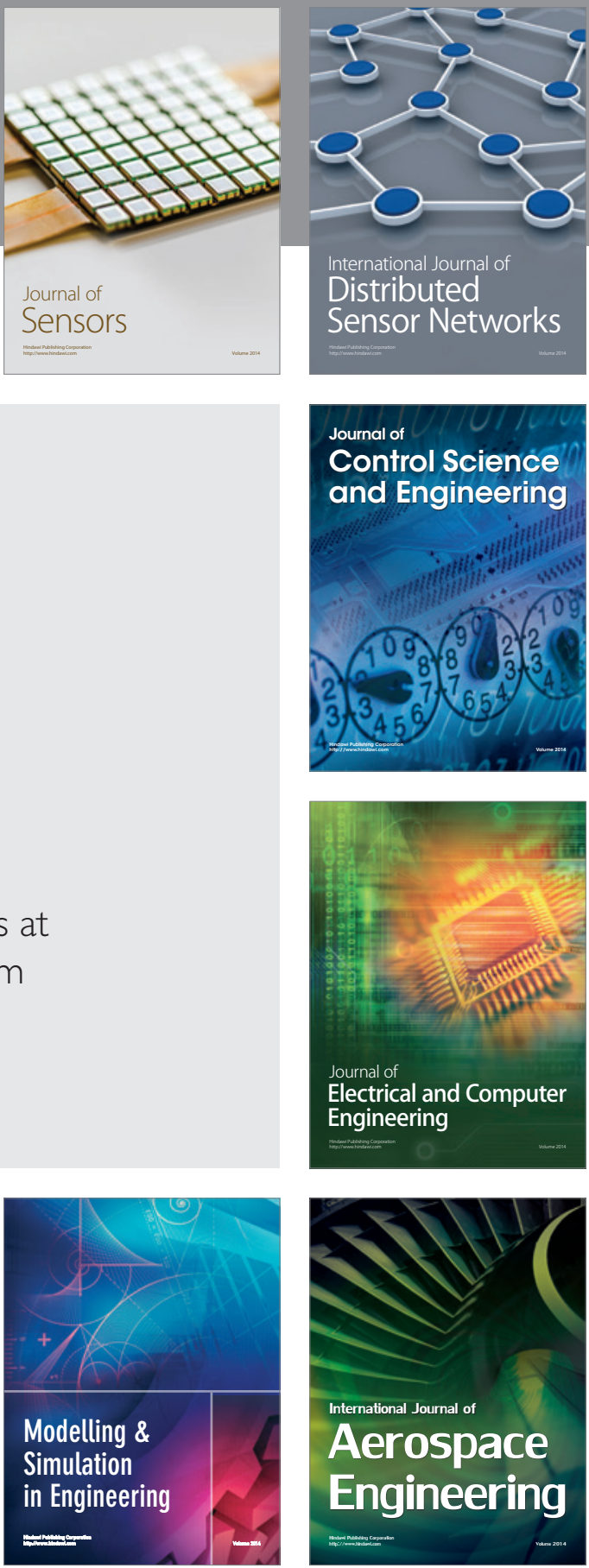

Journal of

Control Science

and Engineering
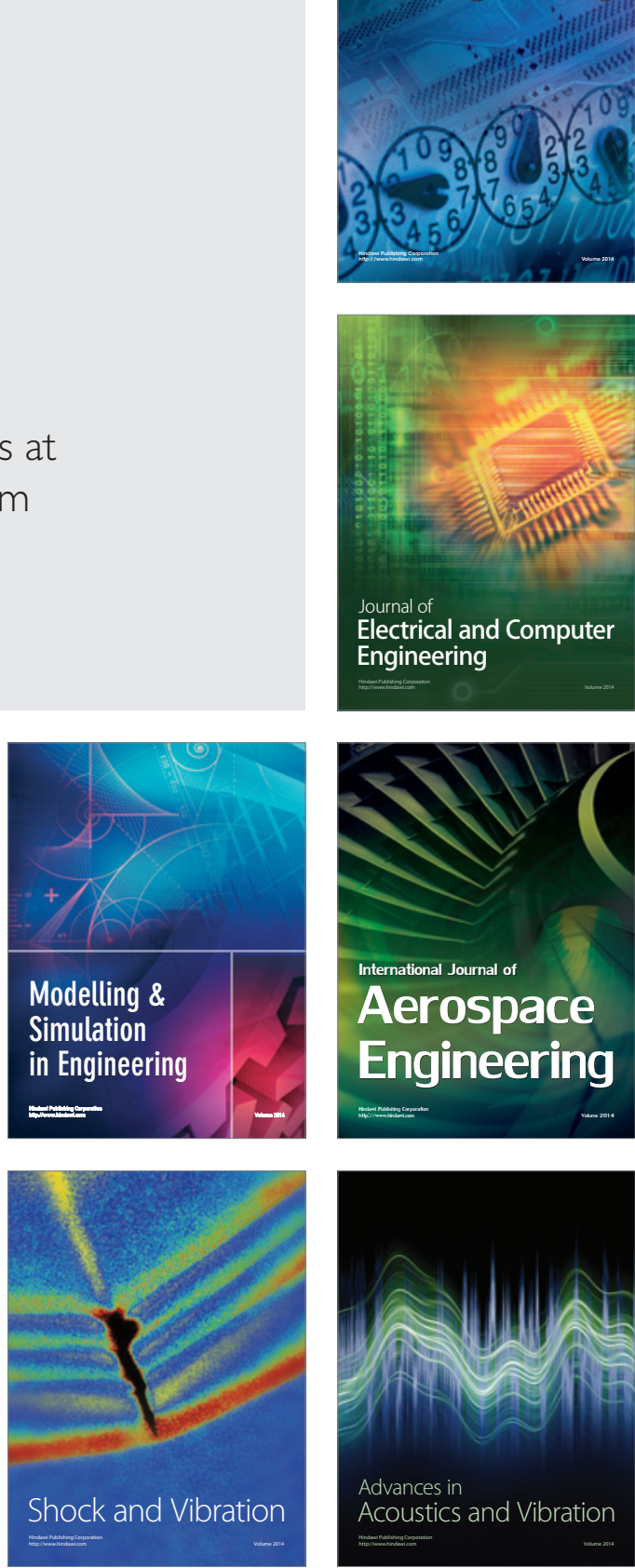